\title{
THE UNIMODALITY OF PURE $O$-SEQUENCES OF TYPE THREE IN THREE VARIABLES
}

\author{
BERNADETTE BOYLE
}

\begin{abstract}
Since the 1970's, great interest has been taken in the study of pure $O$-sequences, which are in bijective correspondence to the Hilbert functions of Artinian level monomial algebras. Much progress has been made in classifying these by their shape. It has been shown that all monomial complete intersections, Artinian algebras in two variables and Artinian level monomial algebras with type two in both three and four variables have unimodal Hilbert functions. This paper proves that Artinian level monomial algebras of type three in three variables have unimodal Hilbert functions. We will also discuss the licciness of these algebras.
\end{abstract}

\section{Introduction}

The study of pure $O$-sequences is a relatively new topic in mathematics as Stanley first introduced them with his paper [24] in 1977. Although pure $O$-sequences are fairly new, they are related to an older algebraic object, Hilbert functions. In particular, they are in bijective correspondence with the Hilbert functions of Artinian level monomial algebras over a polynomial ring $R=k\left[x_{1}, \ldots, x_{n}\right]$ where $k$ is a field. The details of this correspondence will be given in Section 2; we will give some definitions for pure $O$-sequences here.

An order ideal is a non-empty set $X$ of monic monomials such that if $M \in X$ and $N$ is a monomial which divides $M$, then $N \in X$. The $h$-vector of an order ideal is a sequence of numbers which counts the number of monomials in each degree of the order ideal; we denote it as $\underline{h}=\left(h_{0}, h_{1}, \ldots, h_{e}\right)$ with $h_{0}=1$ and $h_{e} \neq 0$. We say that an order ideal is pure if all the maximal monomials have the same degree. A pure $O$-sequence is the $h$-vector of a pure order ideal. The

Received June 18, 2014; received in final form December 17, 2014.

2010 Mathematics Subject Classification. Primary 13D40, 13E10. Secondary 13C40, $13 \mathrm{~F} 20,05 \mathrm{E} 40$. 
type of an $O$-sequence is the number of maximal monomials in the order ideal; if the order ideal is pure, then the type is equal to $h_{e}$. A sequence is unimodal if it does not increase after a strict decrease. A sequence is strictly unimodal if it is unimodal and only constant in its peak degree(s). Pure $O$-sequences and the Hilbert functions of monomial algebras are not affected by the characteristic; therefore without loss of generality, we assume characteristic zero on the field $k$ throughout this paper.

Over the years pure $O$-sequences have inspired a lot of interest in light of their connections with error correcting codes [15], topological combinatorics [5], matroid complexes ([24], [17], [21], [26], [23]), and more. The reader can look at [2] for more examples. In this paper, we will focus our study on the shape of pure $O$-sequences and their connection to Hilbert functions.

One of the first results regarding the shape of pure $O$-sequences is due to Hibi. He showed in [12] (Theorem 1.1) that all pure $O$-sequences, $\underline{h}=$ $\left(1, h_{1}, h_{2}, \ldots, h_{e}\right)$, are flawless. This means that

$$
h_{i} \leq h_{e-i} \quad \text { for all } i \leq\left\lfloor\frac{e}{2}\right\rfloor .
$$

Hibi went on to show that

$$
h_{i-1} \leq h_{i} \quad \text { for all } i \leq\left\lfloor\frac{e}{2}\right\rfloor,
$$

or that the first half of $\underline{h}$ is non-decreasing. Hausel extended this result by showing that the first half of a pure $O$-sequence is differentiable ([11], Theorem 6.3). This means that the first difference of the first half of the pure $O$-sequence satisfies Macaulay's theorem [1], thus, it is the $\underline{h}$-vector of an order ideal. This result was extended by Boij, Migliore, Miro-Roig, Nagel and Zanello when they proved that an $O$-sequence is the first half of a pure $O$-sequence if and only if it is differentiable [2].

In this paper, we are not focusing solely on the first half of the pure $O$ sequence, but rather the whole sequence, asking whether or not it is guaranteed to be unimodal. We do know that there exists non-unimodal pure $O$-sequences. Stanley gave the first example in [24] where he showed that $(1,505,2065,3395,3325,3493)$ is a pure $O$-sequence. In [2] (Theorem 3.9), for any given integers $r \geq 3$ and $M \geq 1$, the authors found that there exists a pure $O$-sequence in $r$ variables which has $M$ maxima. We do not have a complete characterization of which pure $O$-sequences are unimodal, but some partial results have been found by fixing the type and/or the number of variables. Stanley [25], J. Watanabe [27] and Reid, Roberts and Roitman [22] all proved that in any number of variables, all complete intersections have unimodal Hilbert functions. A complete intersection is an ideal generated by a regular sequence. The Hilbert function of a complete intersection corresponds to pure $O$-sequences of type 1 . One tool that was helpful in proving this result is the Weak Lefschetz property (WLP). The Weak Lefschetz property says that 
multiplication by a general linear form from any component of the algebra to the next component has maximal rank. This property forces the sequence to be unimodal due to the standard grading of the algebra. To see this and for more information on the WLP, one can look at [10]. In two variables, Macaulay's theorem [1] immediately implies that all Artinian algebras have unimodal Hilbert functions. In three variables, the authors of [2] showed that all Artinian level monomial algebras of type two have the Weak Lefschetz property and thus have unimodal Hilbert functions (Corollary 6.8). Unfortunately, they also found that the only Artinian level monomial algebras that are guaranteed to have the WLP are those with type one (in any number of variables), those in one or two variables (with any type) and those with type two in three variables ([2], Theorem 7.17). In fact, Brenner and Kaid showed that the WLP can fail for an almost complete intersection (a prime ideal, $P$, which is generated by $h t(P)+1$ generators) with type as low as three [4]. Furthermore, Zanello found a counterexample in [28] of a level Artinian monomial algebra in three variables which fails to have the WLP.

In regard to the shape of pure $O$-sequences, the question has now become whether or not a pure $O$-sequence can be guaranteed to be unimodal even if it does not have the WLP. It was shown in [3] that pure $O$-sequences of type two in four variables are strictly unimodal. The proof relies heavily on the fact that the Hilbert function of a complete intersection peaks in the middle degree. In three variables, which is what we will focus on in this paper, the smallest known type of pure $O$-sequence which fails to be unimodal is fourteen ([2], Example 3.10). This leads one to ask if pure $O$-sequences are guaranteed to be unimodal in three variables with smaller type. In Section 4 of this paper, we will give a positive answer for the smallest open case in three variables, specifically, that pure $O$-sequences of type three in three variables are strictly unimodal. Our proof will use techniques different from those used in previous results. We will decompose the Hilbert functions into complete intersections in two variables and use known information about them.

In Section 3, we will classify all Artinian level monomial algebras of type three in three variables into four classes of ideals which will be used in Sections 4 and 5 of this paper. In Section 5, we will explore the liaison classes of these ideals.

Two ideals $A$ and $B$ are $C I$-linked (respectively G-linked) if there exists a complete intersection ideal $C$ (respectively Gorenstein ideal) such that $C \subseteq$ $A \cap B,[C: A]=B$ and $[C: B]=A$. This is denoted as $A \stackrel{C}{\sim} B$. If two ideals can be linked to each other in a finite number of links, they are in the same liaison class. An ideal is licci if it is in the liaison class of a complete intersection where all the links are complete intersections. Similarly, an ideal is glicci if it is the liaison class of a complete intersection, where all the links are Gorenstein ideals. The liaison classes of ideals are interesting to study since linkage 
preserves several invariants such as codimension, certain cohomology modules, and the property of being arithmetically Cohen-Macaulay. In particular, it is especially nice to study the liaison class of complete intersections. In this section, we will show that two of the four classes of Artinian level monomial algebras of type three in three variables are licci while the other two classes are not, although we conjecture that they are glicci.

\section{Background}

In this paper, we will study pure order ideals and pure $O$-sequences in light of their bijective correspondence with Artinian level monomial algebras and Hilbert functions. Let $R=k\left[x_{1}, \ldots, x_{r}\right]$ with $k$ a field and $I$ a homogeneous monomial ideal of $R$ with no non-zero elements in degree one. We can create a standard graded Artinian monomial $k$-algebra with codimension $r, R / I=$ $\bigoplus_{i \geq 0}(R / I)_{i}$. The Hilbert function of $R / I$ is

$$
H(R / I, i)=\operatorname{dim}_{k}(R / I)_{i}=\operatorname{dim}_{k} R_{i}-\operatorname{dim}_{k} I_{i} .
$$

We know that the Hilbert function of an Artinian ideal is necessarily finite, thus we can denote the Hilbert function as $H(R / I)=\left(h_{0}=1, h_{1}, h_{2}, \ldots, h_{e}\right)$, where $e$ is the last degree $i$ for which $H(R / I, i) \neq 0\left(h_{i}>0\right.$ for $\left.0 \leq i \leq e\right)$. If $\mathfrak{m}=\left(x_{1}, \ldots, x_{r}\right)$, the maximal ideal of $R$, then the homogeneous maximal ideal in $R / I$ is $\overline{\mathfrak{m}}=\left(\overline{x_{1}}, \ldots, \overline{x_{r}}\right)$. We call the annihilator of $\overline{\mathfrak{m}}$ the socle of $R / I$ so $\operatorname{soc}(R / I)=\{a \in R / I \mid a \overline{\mathfrak{m}}=0\}$. Since our algebras are level, the socle is entirely contained in degree $e$, which we call the socle degree. The type of $R / I$ is the dimension of the socle, which is equal to $h_{e}$ since our algebras are level.

We note that order ideals are closed by division on the monomials and ring ideals are closed by taking multiples. Thus, it is clear that in each degree $d$, the ring ideal contains the exact monomials which are not in the order ideal and vice versa. This can been shown more formally using Macaulay's theory of inverse systems where the collection of monomials in $R_{d}$ that are not in $I_{d}$ is the inverse system to $I$. One can learn more about Macaulay's theory of inverse systems in [7], [8] or the Appendix of [14]. When we translate between order ideals and Artinian monomial algebras in this way, many properties are preserved. The order ideal is pure if and only if the related Artinian monomial algebra is level. Also, the type of the order ideal is the same as the type of the algebra. Finally, we know that the Hilbert function of an Artinian level monomial algebra is equal to the related pure $O$-sequence, thus they are bijective correspondence.

We will now discuss some results in liaison theory that will be used later in this paper. The study of liaison theory has led to the construction of particular ideals, such as basic double links and liaison addition. Let $J \subset$ $I \subset R=k\left[x_{1}, \ldots, x_{r}\right]$ where $J$ and $I$ are homogeneous ideals with $\operatorname{codim}(J)=$ $\operatorname{codim}(I)-1$. Let $f \in R$ be homogeneous, with $J: f=J$. Then $I^{\prime}:=f \cdot I+J$ is 
a basic double link. This name comes from the fact that $I^{\prime}$ can be Gorenstein linked to $I$ in two steps if $I$ is unmixed. $I$ is constructed from liaison addition if $I=F_{1} \cdot I_{1}+F_{2} \cdot I_{2}+\cdots+F_{k} \cdot I_{k}$ where $\left(F_{1}, F_{2}, \ldots, F_{k}\right)$ is a regular sequence and $F_{i} \in \bigcap_{j \neq i, 1 \leq j \leq k} I_{j}$. For each $j, F_{j}$ is in $R=k\left[x_{1}, \ldots, x_{r}\right]$ and $I_{j}$ is a homogeneous ideal or $R$. There has been much progress studying liaison, but we will only state the known results that will be needed in the rest of the paper, namely the Hilbert function formula. We refer the reader to [18] for more information on the full construction of these theories.

Lemma 2.1. Let $R=k\left[x_{1}, \ldots, x_{r}\right]$ and $J$ and $I$ be homogeneous ideals so $J \subset I \subset R$. Let $f \in R, \operatorname{deg}(f)=d$ and $J: f=J$. Then for $I^{\prime}:=f \cdot I+J$, and each integer $t$, we have

$$
H\left(R / I^{\prime}, t\right)=H(R / J, t)+H(R / I, t-d)-H(R / J, t-d) .
$$

In particular, if $J$ is the complete intersection $\left(x_{1}^{a_{1}}, x_{2}^{a_{2}}, \ldots, \widehat{x}_{i}^{a_{i}}, \ldots, x_{k}^{a_{k}}\right)$ and $f=x_{i}^{a_{i}}$, then

$$
H\left(R / I^{\prime}, t\right)=H(R / \tilde{J}, t)+H\left(R / I, t-a_{i}\right),
$$

where $\tilde{J}=\left(x_{1}^{a_{1}}, x_{2}^{a_{2}}, \ldots, \widehat{x_{i}^{a_{i}}}, \ldots, x_{k}^{a_{k}}, f\right)=\left(x_{1}^{a_{1}}, x_{2}^{a_{2}}, \ldots, x_{k}^{a_{k}}\right)$.

Proof. We have the exact sequence

$$
0 \longrightarrow J(-d) \longrightarrow J \oplus I(-d) \longrightarrow I^{\prime} \longrightarrow 0 .
$$

This sequence gives us

$$
\operatorname{dim}\left(I^{\prime}\right)_{t}=\operatorname{dim}(I)_{t-d}+\operatorname{dim}(J)_{t}-\operatorname{dim}(J)_{t-d} .
$$

From this equality, we get

$$
H\left(R / I^{\prime}, t\right)=H(R / J, t)+H(R / I, t-d)-H(R / J, t-d) .
$$

For the complete intersection, the Hilbert function follows since

$$
H(R / \tilde{J}, t)=H(R / J, t)-H\left(R / J, t-a_{i}\right) .
$$

Lemma 2.2. For each $j$, let $I_{j}$ be a homogeneous ideal (or the whole ring) and $F_{j} \in R=k\left[x_{1}, x_{2}, \ldots, x_{r}\right]$ where $\operatorname{deg}\left(F_{j}\right)=d_{j}$. Also, let $\left(F_{1}, F_{2}, \ldots, F_{k}\right)$ be a regular sequence with $F_{i} \in \bigcap_{j \neq i, 1 \leq j \leq k} I_{j}$. Then for $I=F_{1} \cdot I_{1}+F_{2} \cdot I_{2}+$ $\cdots+F_{k} \cdot I_{k}$ and $L=\left(F_{1}, F_{2}, \ldots, F_{k}\right)$ we have

$$
\begin{aligned}
H(R / I, t)= & H(R / L, t)+H\left(R / I_{1}, t-d_{1}\right)+H\left(R / I_{2}, t-d_{2}\right) \\
& +\cdots+H\left(R / I_{k}, t-d_{k}\right) .
\end{aligned}
$$

This result is very similar to results in earlier papers including [9] Theorem 1.3 and [20] Proposition 4.1. To prove this, mimic the proof given in [9].

We will end this section introducing some notation that will be used, as well as some lemmas and remarks which will be referenced in the paper. 
Definition 2.3. $H\left(a_{1}, \ldots, a_{r}\right)$ will denote the Hilbert function of an Artinian complete intersection with the form $\left(x_{1}^{a_{1}}, x_{2}^{a_{2}}, \ldots, x_{r}^{a_{r}}\right)$ in $k\left[x_{1}, \ldots, x_{r}\right]$ $(r \geq 1)$.

Lemma 2.4. Let $\mathfrak{a}=\left(x_{1}^{a_{1}}, x_{2}^{a_{2}}, \ldots, x_{r}^{a_{r}}\right)$ be a complete intersection in the ring $k\left[x_{1}, \ldots, x_{r}\right]$. Let $\Delta H$ be the first difference of its Hilbert function. Then

$$
\Delta H=H\left(a_{1}, a_{2}, \ldots, a_{r-1}\right)-H\left(a_{1}, a_{2}, \ldots, a_{r-1}\right)\left(-a_{r}\right),
$$

where $H\left(a_{1}, a_{2}, \ldots, a_{r-1}\right)$ and $H\left(a_{1}, a_{2}, \ldots, a_{r-1}\right)\left(-a_{r}\right)$ are in the ring $k\left[x_{1}, \ldots, x_{r-1}\right]$. Any permutation of the $a_{i}$ is equally valid.

In this paper, we will primarily use Lemma 2.4 with $r=3$.

Remark 2.5 (Theorem 1 [22] and Theorem 0.4 [1]). The first difference of a Hilbert function of a complete intersection in two variables with a shift of $s$ is:

$$
\Delta H(a, b)(-s) \text { in degree } t= \begin{cases}0 & \text { until } t \geq s \\ 1 & \text { until } t \geq s+\text { the minimum of }\{a, b\} \\ 0 & \text { until } t \geq s+\text { the maximum of }\{a, b\} \\ -1 & \text { until } t \geq a+b+s \\ 0 & \text { for } t \geq a+b+s .\end{cases}
$$

\section{Classification theorem}

We will classify the Hilbert functions of Artinian level monomial algebras of type three in three variables into four classes.

TheOREM 3.1. Let $R=k[x, y, z]$ and let $I$ be a monomial ideal such that $R / I$ is Artinian and level of type three. Then I has one of the following four forms, up to a change of variables. (Without loss of generality, we will assume that $a \geq \alpha_{2} \geq \alpha_{1}, b \geq \beta_{2} \geq \beta_{1}, c \geq \gamma_{2} \geq \gamma_{1}$.)

(1) $\left(x^{a}, x^{\alpha_{2}} z^{\gamma_{1}}, x^{\alpha_{1}} z^{\gamma_{2}}, z^{c}, y^{\beta_{1}} z^{\gamma_{2}}, y^{\beta_{2}} z^{\gamma_{1}}, y^{b}\right)$ where $a+b+\gamma_{1}=\alpha_{2}+\beta_{2}+\gamma_{2}=$ $\alpha_{1}+\beta_{1}+c$. The Hilbert function of $R / I$ is

$$
H\left(a, b, \gamma_{1}\right)+H\left(\alpha_{2}, \beta_{2}, \gamma_{2}-\gamma_{1}\right)\left(-\gamma_{1}\right)+H\left(\alpha_{1}, \beta_{1}, c-\gamma_{2}\right)\left(-\gamma_{2}\right) .
$$

(2) $\left(x^{a}, x^{\alpha_{2}} z^{\gamma_{1}}, x^{\alpha_{1}} z^{\gamma_{2}}, z^{c}, y^{\beta_{2}} z^{\gamma_{1}}, y^{b}, x^{\alpha_{1}} y^{\beta_{1}} z^{\gamma_{1}}\right)$ where $a+b+\gamma_{1}=\alpha_{2}+\beta_{1}+$ $\gamma_{2}=\alpha_{1}+\beta_{2}+c$. The Hilbert function of $R / I$ is

$$
H\left(a, b, \gamma_{1}\right)+H\left(\alpha_{1}, \beta_{2}, c-\gamma_{1}\right)\left(-\gamma_{1}\right)+H\left(\alpha_{2}-\alpha_{1}, \beta_{1}, \gamma_{2}-\gamma_{1}\right)\left(-\alpha_{1}-\gamma_{1}\right) .
$$

(3) $\left(x^{a}, x^{\alpha_{2}} z^{\gamma_{1}}, x^{\alpha_{2}} y^{\beta_{1}}, z^{c}, y^{\beta_{2}} z^{\gamma_{2}}, x^{\alpha_{1}} y^{\beta_{2}}, y^{b}\right)$ where $a+\beta_{1}+\gamma_{1}=\alpha_{2}+\beta_{2}+c=$ $\alpha_{1}+b+\gamma_{2}$. The Hilbert function of $R / I$ is

$$
H\left(\alpha_{2}, \beta_{2}, c\right)+H\left(a-\alpha_{2}, \beta_{1}, \gamma_{1}\right)\left(-\alpha_{2}\right)+H\left(\alpha_{1}, b-\beta_{2}, \gamma_{2}\right)\left(-\beta_{2}\right) .
$$


(4) $\left(x^{a}, x^{\alpha_{2}} z^{\gamma_{1}}, z^{c}, y^{\beta_{1}} z^{\gamma_{2}}, y^{b}, x^{\alpha_{1}} y^{\beta_{2}}, x^{\alpha_{1}} y^{\beta_{1}} z^{\gamma_{1}}\right)$ where $a+\beta_{2}+\gamma_{1}=\alpha_{1}+b+$ $\gamma_{2}=\alpha_{2}+\beta_{1}+c$. The Hilbert function of $R / I$ is

$$
\begin{aligned}
& H\left(\alpha_{1}, b, \gamma_{1}\right)+H\left(a-\alpha_{1}, \beta_{2}, \gamma_{1}\right)\left(-\alpha_{1}\right)+H\left(\alpha_{2}, \beta_{1}, c-\gamma_{1}\right)\left(-\gamma_{1}\right) \\
& \quad+H\left(\alpha_{1}, b-\beta_{1}, \gamma_{2}-\gamma_{1}\right)\left(-\beta_{1}-\gamma_{1}\right) .
\end{aligned}
$$

Proof. A monomial Artinian level algebra of type three over $k[x, y, z]$ is the pure $O$-sequence generated by three monomials of the same degree. Let the monomials be: $x^{r_{1}} y^{s_{1}} z^{t_{1}}, x^{r_{2}} y^{s_{2}} z^{t_{2}}$, and $x^{r_{3}} y^{s_{3}} z^{t_{3}}$ where $r_{1}+s_{1}+t_{1}=$ $r_{2}+s_{2}+t_{2}=r_{3}+s_{3}+t_{3}$.

Using the fact that the three monomials must have the same degree, we can find all possible cases by evaluating the inequalities between the exponents. In doing so, we find that there are, up to change of variables, four possible cases. Case 1: each monomial has one smallest exponent (either $\min \left\{r_{i}\right\}$, $\min \left\{s_{i}\right\}$ or $\min \left\{t_{i}\right\}$ ), one middle exponent and one largest exponent. Case 2: one monomial has all three middle exponents, another monomial has two of the smallest exponents and one largest exponent and the final monomial has the two remaining largest exponents and one smallest exponent. Case 3: one monomial has two of the largest exponents and one smallest exponent, another monomial has two middle exponents and one smallest exponent and the final monomial has the remaining smallest, middle and largest exponent. Case 4: one monomial has two of the smallest exponents and one largest exponent, another monomial has two middle exponents and one largest exponent and the final monomial has the remaining smallest, middle and largest exponent.

Now let us look at the corresponding ideals in each case.

Case 1: Up to change of variables, case 1 can be given by $r_{3} \geq r_{2} \geq r_{1}$, $s_{1} \geq s_{3} \geq s_{2}$ and $t_{2} \geq t_{1} \geq t_{3}$ (with $t_{3} \neq t_{2}$ ). The ring ideal generated the monomials not in the pure $O$-sequence is

$$
I=\left(x^{r_{3}+1}, y^{s_{1}+1}, z^{t_{2}+1}, x^{r_{2}+1} z^{t_{3}+1}, x^{r_{1}+1} y^{s_{3}+1}, y^{s_{2}+1} z^{t_{1}+1}, x^{r_{1}+1} y^{s_{2}+1} z^{t_{3}+1}\right) .
$$

To match the notation of the proposition, rename the variables so that greatest $x$ exponent is $a$, the second greatest $x$ exponent is $\alpha_{2}$, and the least $x$ exponent is $\alpha_{1}$ and likewise for $y$ and $z$ so that we have the following: $r_{3}+1=a, r_{2}+1=\alpha_{2}, r_{1}+1=\alpha_{1}, s_{1}+1=b, s_{3}+1=\beta_{2}, s_{2}+1=\beta_{1}$, $t_{2}+1=c, t_{1}+1=\gamma_{2}, t_{3}+1=\gamma_{1}$. After these changes, it is clear that this is the ideal (4) above.

Case 2: Up to change of variables, case 2 can be given by $r_{3} \geq r_{2} \geq r_{1}$, $s_{3} \geq s_{2} \geq s_{1}$ and $t_{1}>t_{2}>t_{3}$. The ring ideal generated the monomials not in the pure $O$-sequence is

$$
I=\left(x^{r_{3}+1}, y^{s_{3}+1}, z^{t_{1}+1}, x^{r_{2}+1} z^{t_{3}+1}, y^{s_{2}+1} z^{t_{3}+1}, x^{r_{1}+1} z^{t_{2}+1}, y^{s_{1}+1} z^{t_{2}+1}\right) .
$$

After properly renaming the variables, we have the ideal (1) above. 
Case 3: Up to change of variables, case 3 can be given by $r_{3} \geq r_{2} \geq r_{1}$, $s_{3} \geq s_{1} \geq s_{2}$ and $t_{1} \geq t_{2}>t_{3}$. The ring ideal generated the monomials not in the pure $O$-sequence is

$$
I=\left(x^{r_{3}+1}, y^{s_{3}+1}, z^{t_{1}+1}, y^{s_{1}+1} z^{t_{3}+1}, x^{r_{2}+1} z^{t_{3}+1}, x^{r_{1}+1} z^{t_{2}+1}, x^{r_{1}+1} y^{s_{2}+1} z^{t_{3}+1}\right) .
$$

After properly renaming the variables, we have the ideal (2) above.

Case 4: Up to change of variables, case 4 can be given by $r_{3} \geq r_{2} \geq r_{1}$, $s_{1} \geq s_{2} \geq s_{3}$ and $t_{2} \geq t_{1}>t_{3}$. The ring ideal generated the monomials not in the pure $O$-sequence is

$$
I=\left(x^{r_{3}+1}, y^{s_{1}+1}, z^{t_{2}+1}, x^{r_{2}+1} y^{s_{3}+1}, x^{r_{1}+1} y^{s_{2}+1}, x^{r_{2}+1} z^{t_{3}+1}, y^{s_{2}+1} z^{t_{1}+1}\right) .
$$

After properly renaming the variables, we have the ideal (3) above.

Now we will show that each ideal has the respective Hilbert function. Ideal (1), decomposes as

$$
z^{\gamma_{1}} \cdot L+\left(x^{a}, y^{b}\right), \quad \text { where } L=z^{\gamma_{2}-\gamma_{1}}\left(x^{\alpha_{1}}, y^{\beta_{1}}, z^{c-\gamma_{2}}\right)+\left(x^{\alpha_{2}}, y^{\beta_{2}}\right) .
$$

Similarly, ideal (2) equals

$$
z^{\gamma_{1}} \cdot L+\left(x^{a}, y^{b}\right), \quad \text { where } L=x^{\alpha_{1}}\left(x^{\alpha_{2}-\alpha_{1}}, y^{\beta_{1}}, z^{\gamma_{2}-\gamma_{1}}\right)+\left(y^{\beta_{2}}, z^{c-\gamma_{1}}\right) .
$$

For both these ideals, the Hilbert function follows by using the formula of Lemma 2.1 twice.

Ideal (3), decomposes as

$$
x^{\alpha_{2}} \cdot\left(x^{a-\alpha_{2}}, y^{\beta_{1}}, z^{\gamma_{1}}\right)+y^{\beta_{2}} \cdot\left(x^{\alpha_{1}}, y^{b-\beta_{2}}, z^{\gamma_{2}}\right)+z^{c} .
$$

The Hilbert function follows by using the formula given in Lemma 2.2.

Ideal (4) decomposes as

$$
\begin{aligned}
I & =z^{\gamma_{1}}\left[y^{\beta_{1}}\left(x^{\alpha_{1}}, y^{b-\beta_{1}}, z^{\gamma_{2}-\gamma_{1}}\right)+\left(x^{\alpha_{2}}, z^{c-\gamma_{1}}\right)\right]+\left(x^{a}, x^{\alpha_{1}} y^{\beta_{2}}, y^{b}\right) \\
& =z^{\gamma_{1}} \cdot L+J .
\end{aligned}
$$

In this case, the Hilbert function is a result of the formula found in Lemma 2.1 applied multiple times. First, it is used to find the Hilbert function of $L$. Then it is used it to find the Hilbert function of

$$
\left(J, z^{\gamma_{1}}\right)=\left(x^{a}, x^{\alpha_{1}} y^{\beta_{1}}, y^{b}, z^{\gamma_{1}}, x^{\alpha_{1}} z^{\gamma_{1}}\right)=x^{\alpha_{1}}\left(x^{a-\alpha_{1}}, y^{\beta_{1}}, z^{\gamma_{1}}\right)+\left(y^{b}, z^{\gamma_{1}}\right) .
$$

Finally, it is used one more time to find the Hilbert function of $I=z^{\gamma_{1}} \cdot L+J$, which gives the desired result.

\section{Main theorem}

ThEOREM 4.1. Let $R=k[x, y, z]$ and let $I$ be a monomial Artinian ideal such that $R / I$ is level of type three. The Hilbert function of $R / I$ is strictly unimodal. 
This result generalizes the result of Cook and Nagel ([6], Theorem 5.4) which says level Artinian monomial almost complete intersections in three variables have strictly unimodal Hilbert functions.

Before beginning the proof of the theorem, we will comment on the notation level level

$\leq$ and $\geq$. These mean that the particular inequality is true from the levelness of the ideal. For example in Case 1 , for levelness we need $a+b+\gamma_{1}=$ $\alpha_{2}+\beta_{2}+\gamma_{2}=\alpha_{1}+\beta_{1}+c$. Thus, since $\beta_{2} \geq \beta_{1}$ and $\alpha_{2}+\beta_{2}+\gamma_{2}=\alpha_{1}+\beta_{1}+c$, we have that $\alpha_{1}+c \geq \alpha_{2}+\gamma_{2}$.

Theorem 3.1 says that it is enough to show the unimodality for the Hilbert functions of the four classes found in Section 3. The first two cases have Hilbert functions that break down using basic double linkage, thus a similar argument will work for both; these two ideals will be addressed in Proposition 4.2. The Hilbert function of the third case breaks down according to liaison addition, and the shifts line up differently from the first two ideals. The Hilbert function of the fourth case breaks into four pieces instead of three like the other cases. Thus, the third and fourth ideals will be addressed independently in Propositions 4.4 and 4.6, respectively.

Proposition 4.2. Let

$$
I_{1}=\left(x^{a}, x^{\alpha_{2}} z^{\gamma_{1}}, x^{\alpha_{1}} z^{\gamma_{2}}, z^{c}, y^{\beta_{1}} z^{\gamma_{2}}, y^{\beta_{2}} z^{\gamma_{1}}, y^{b}\right),
$$

where $a+b+\gamma_{1}=\alpha_{2}+\beta_{2}+\gamma_{2}=\alpha_{1}+\beta_{1}+c$ and $a \geq \alpha_{2} \geq \alpha_{1}, b \geq \beta_{2} \geq \beta_{1}$, $c \geq \gamma_{2} \geq \gamma_{1}$. Let $H_{R / I_{1}}$ be the Hilbert function of $R / I_{1}$, so

$$
H_{R / I_{1}}=H\left(a, b, \gamma_{1}\right)+H\left(\alpha_{2}, \beta_{2}, \gamma_{2}-\gamma_{1}\right)\left(-\gamma_{1}\right)+H\left(\alpha_{1}, \beta_{1}, c-\gamma_{2}\right)\left(-\gamma_{2}\right) .
$$

Similarly, let

$$
I_{2}=\left(x^{a}, x^{\alpha_{2}} z^{\gamma_{1}}, x^{\alpha_{1}} z^{\gamma_{2}}, z^{c}, y^{\beta_{2}} z^{\gamma_{1}}, x^{\alpha_{1}} y^{\beta_{1}} z^{\gamma_{1}}, y^{b}\right),
$$

where $a+b+\gamma_{1}=\alpha_{2}+\beta_{1}+\gamma_{2}=\alpha_{1}+\beta_{2}+c$ and $a \geq \alpha_{2} \geq \alpha_{1}, b \geq \beta_{2} \geq \beta_{1}$, $c \geq \gamma_{2} \geq \gamma_{1}$. Let $H_{R / I_{2}}$ be the Hilbert function of $R / I_{2}$, so

$$
\begin{aligned}
H_{R / I_{2}}= & H\left(a, b, \gamma_{1}\right)+H\left(\alpha_{1}, \beta_{2}, c-\gamma_{1}\right)\left(-\gamma_{1}\right) \\
& +H\left(\alpha_{2}-\alpha_{1}, \beta_{1}, \gamma_{2}-\gamma_{1}\right)\left(-\alpha_{1}-\gamma_{1}\right) .
\end{aligned}
$$

These Hilbert functions are strictly unimodal.

Proof. For strict unimodality, it is enough to show that $\Delta H_{R / I_{i}}$ is positive, then possibly zero, then negative. To simplify the two cases into one, define the following variables.

For $I_{1}$ :

$$
\begin{aligned}
& \rho=\min \{a, b\}, \quad \eta=\min \left\{\alpha_{2}, \beta_{2}\right\}, \quad \nu=\min \left\{\alpha_{1}, \beta_{1}\right\}, \\
& \sigma=\max \{a, b\}, \quad \mu=\max \left\{\alpha_{2}, \beta_{2}\right\}, \quad \omega=\max \left\{\alpha_{1}, \beta_{1}\right\}, \\
& \tau=\gamma_{1}, \quad \kappa=\gamma_{2}-\gamma_{1}, \quad \xi=c-\gamma_{2} .
\end{aligned}
$$


For $I_{2}$ :

$$
\begin{aligned}
& \rho=\min \{a, b\}, \quad \eta=\min \left\{\beta_{2}, c-\gamma_{1}\right\}, \quad \nu=\min \left\{\beta_{1}, \gamma_{2}-\gamma_{1}\right\}, \\
& \sigma=\max \{a, b\}, \quad \mu=\max \left\{\beta_{2}, c-\gamma_{1}\right\}, \quad \omega=\max \left\{\beta_{1}, \gamma_{2}-\gamma_{1}\right\}, \\
& \tau=\gamma_{1}, \quad \kappa=\alpha_{1}, \quad \xi=\alpha_{2}-\alpha_{1} .
\end{aligned}
$$

For both ideals, the decomposition of Lemma 2.4 gives us that

$$
\begin{aligned}
\Delta H= & {[H(\rho, \tau)+H(\eta, \kappa)(-\tau)+H(\nu, \xi)(-\kappa-\tau)] } \\
& -[H(\rho, \tau)(-\sigma)+H(\eta, \kappa)(-\mu-\tau)+H(\nu, \xi)(-\omega-\kappa-\tau)] \\
:= & P-N .
\end{aligned}
$$

For convenience, write this decomposition as

$$
\Delta H_{R / I_{1}}=\left[H_{1}^{+}+H_{2}^{+}+H_{3}^{+}\right]-\left[H_{1}^{-}+H_{2}^{-}+H_{3}^{-}\right] .
$$

We will show that there do not exist integers $t_{1}<t_{2} \leq e$ such that $P\left(t_{1}\right)=$ $N\left(t_{1}\right)$ and $P\left(t_{2}\right)>N\left(t_{2}\right)$ or $P\left(t_{1}\right)<N\left(t_{1}\right)$ and $P\left(t_{2}\right) \geq N\left(t_{2}\right)$. Note that each segment in $N$ is a complete intersection with a corresponding segment in $P$, such that $H_{i}^{-}$and $H_{i}^{+}$have the same strictly unimodal Hilbert function, with the negative piece beginning later.

Claim 1. If $0 \neq P\left(t_{1}\right) \leq N\left(t_{1}\right)$, then $P$ is either constant or decreasing for all degrees $t \geq t_{1}$. Equivalently, $\Delta P(t) \leq 0$ for $t \geq t_{1}$.

Proof. We recall that each segment in $P$ is a complete intersection and has the form given in Remark 2.5. If each $H_{i}^{+}$has not started or is increasing then $P>N$ (or both are 0 ) since $H_{i}^{+} \geq H_{i}^{-}$for each $i$. Thus if $0 \neq P\left(t_{1}\right) \leq N\left(t_{1}\right)$ then at least one positive segment is constant, decreasing or over for all $t \geq t_{1}$.

First, we will check when at least one segment of $P$ is either decreasing or over for all degrees $t \geq t_{1}$. Due to the shifts, no two $\Delta H_{i}^{+}$can be positive in the same degree. Thus if $\Delta P(t)$ is positive for some $t \geq t_{1}$, we need at least one of the $\Delta H_{i}^{+}(t)$ to equal one, another to have ended, and the third cannot equal -1 . However, this cannot occur:

(1) If $H_{3}^{+}(t)$ is over, then $t \geq \nu+\xi+\kappa+\tau \geq \kappa+\tau$ so $\Delta H_{1}^{+} \neq 1$ and $\Delta H_{2}^{+} \neq 1$.

(2) If $H_{2}^{+}(t)$ is over, then $t \geq \eta+\kappa+\tau \geq \nu+\kappa+\tau$ so $\Delta H_{1}^{+} \neq 1$ and $\Delta H_{3}^{+} \neq 1$.

(3) Now say $H_{1}^{+}(t)$ has ended and look at $I_{1}$ and $I_{2}$ separately. For $I_{2}$, if $\rho=a$ then $t \geq \rho+\tau=a+\tau \geq \xi+\kappa+\tau \geq \kappa+\tau$, so neither $H_{2}^{+}$nor $H_{3}^{+}$ equal 1, so this subcase is done. Now we can combine the case for $I_{1}$ $(\rho=a$ or $b)$ with the case for $I_{2}$ where $\rho=b$. If $H_{1}^{+}(t)$ has ended, then $t \geq \rho+\tau \geq \eta+\tau$ which implies that $\Delta H_{2}^{+}(t)$ cannot equal 1 ; therefore it remains to check when $\Delta H_{3}^{+}(t)=1$, so $t \geq \kappa+\tau$. Together the inequalities $t \geq \kappa+\tau$ and $t \geq \eta+\tau$ imply that $\Delta H_{2}^{+}(t)=-1$; therefore, $\Delta P(t) \leq 0$.

We have shown that if any segment of $P\left(t_{1}\right)$ is decreasing or over, then $\Delta P(t) \leq 0$ for $t \geq t_{1}$. Now, we will look at the case with $P\left(t_{1}\right) \leq N\left(t_{1}\right)$ when some segment(s) of $P$ are constant in degree $t_{1}$. We can assume that the 
other segments of $P$ are not decreasing or over as that is addressed above. Furthermore, we cannot have a segment of $P$ be increasing in degree $t_{1}$, since then $P\left(t_{1}\right)>N\left(t_{1}\right)$, contradicting our assumption. If all three segments of $P$ are constant in degree $t_{1}$, then none of them can increase for $t \geq t_{1}$ and $\Delta P(t) \leq 0$ for all $t \geq t_{1}$. If two segments, $H_{2}^{+}\left(t_{1}\right)$ and $H_{1}^{+}\left(t_{1}\right)$, are constant (and $H_{3}^{+}\left(t_{1}\right)$ not started), then $t_{1} \geq \eta+\tau \geq \tau$. For $P\left(t_{1}\right) \leq N\left(t_{1}\right)$, we need $H_{1}^{-}\left(t_{1}\right)$ to be constant so $t_{1} \geq \tau+\sigma>\sigma \geq \rho$ or $t_{1} \geq \rho+\sigma>\rho$. Since $t_{1} \geq \tau$ and $t_{1} \geq \rho$, we have that $H_{1}^{+}\left(t_{1}\right)$ is decreasing or over, which addressed above. Finally let $P\left(t_{1}\right) \geq N\left(t_{1}\right)$ with $H_{1}^{+}\left(t_{1}\right)$ constant and $H_{2}^{+}\left(t_{1}\right)$ and $H_{3}^{+}\left(t_{1}\right)$ not yet started, thus $t_{1}<\tau$ and $t_{1} \geq \rho$. If $P(t)$ increases for $t \geq t_{1}$, then $H_{2}^{+}(t)$ needs to be increasing which means that $t \geq \tau$. Thus, $H_{1}^{+}(t)$ simultaneously starts decreasing when $\mathrm{H}_{2}^{+}$starts increasing, so $P(t)$ is still constant. Since $\mathrm{H}_{1}^{+}$is now decreasing, the rest of this case is address above.

Claim 2. There do not exist degrees $t_{1}<t_{2} \leq e$ such that $P\left(t_{1}\right)=N\left(t_{1}\right)$ and $N\left(t_{2}\right)<P\left(t_{2}\right)$ or $P\left(t_{1}\right)<N\left(t_{1}\right)$ and $N\left(t_{2}\right) \leq P\left(t_{2}\right)$.

Proof. Claim 1 says that $P$ will not increase after $t_{1}$ and thus if such a $t_{2}$ exists, then $N$ needs to decrease faster than $P$. We will examine when each segment of $N$ is decreasing to show that such a $t_{2}$ does not exist. Before doing so, we remark on the relationship between the segments of $P$ and $N$.

REMARK 4.3. (i) If $\Delta H_{i}^{-}(t)=-1$, then $H_{i}^{+}$has ended in some degree less than or equal to $t$. We see this since,

$$
\begin{array}{lll}
\Delta H_{1}^{-}(t)=-1 \quad & \Rightarrow t \geq \tau+\sigma \geq \tau+\rho \Rightarrow H_{1}^{+} \text {ended, } \\
\Delta H_{2}^{-}(t)=-1 \quad \Rightarrow \quad t \geq \kappa+\mu+\tau \geq \kappa+\eta+\tau \Rightarrow H_{2}^{+} \text {ended, } \\
\Delta H_{3}^{-}(t)=-1 \quad \Rightarrow \quad t \geq \xi+\omega+\kappa+\tau \geq \xi+\nu+\kappa+\tau \Rightarrow H_{3}^{+} \text {ended. }
\end{array}
$$

(ii) If $\Delta H_{j}^{-}(t)=-1$ for some $j$, then $H_{i}^{+}(s)<H_{j}^{-}(s)$ for $s \geq t$ and all $i$. To see this, we note that since the ideal is level of type three, then when $H_{j}^{-}(t)$ ends, all the three of the segments of $N$ also end and the whole Hilbert function ends. Furthermore, no segments except the three $H_{j}^{-}$can be non-zero in this last degree; in particular all the $H_{i}^{+}$must end before any $H_{j}^{-}$ends. $H_{i}^{+}$cannot decrease by more than one each degree, so if $H_{j}^{-}$is decreasing by one each degree and ends after $H_{i}^{+}$, then $H_{i}^{+}(s)<H_{j}^{-}(s)$ for all $s \geq t$.

Now we will look at when each segment of $N$ is decreasing with $t \geq t_{1}$.

(1) $\Delta H_{3}^{-}(t)=-1$. Remark 4.3 says $H_{3}^{+}(t)$ is over. $H_{2}^{+}(t)$ is over since for $I_{1}: \quad t \geq \omega+\kappa+\tau+\xi=\omega+c \geq \alpha_{1}+c \stackrel{\text { level }}{\geq} \alpha_{2}+\gamma_{2} \geq \eta+\kappa+\tau$, for $I_{2}: \quad t \geq \omega+\kappa+\tau+\xi \geq \beta_{1}+\alpha_{2}+\gamma_{1} \stackrel{\text { level }}{\geq} \alpha_{1}+\beta_{2}+\gamma_{1} \geq \eta+\kappa+\tau$. 
Thus, $P(t)=H_{1}^{+}(t)$ and Remark 4.3 implies that $H_{3}^{-}(s)>H_{1}^{+}(s)$ so $N(s)>P(s)$ for $s \geq t$.

(2) $\Delta H_{2}^{-}(t)=-1$. Remark 4.3 says $H_{2}^{+}(t)$ is over. $H_{1}^{+}(t)$ is over since

$$
\begin{array}{ll}
\text { for } I_{1}: & t \geq \mu+\tau+\kappa \geq \alpha_{2}+\gamma_{2} \stackrel{\text { level }}{\geq} a+\gamma_{1} \geq \rho+\tau, \\
\text { for } I_{2}: & t \geq \mu+\tau+\kappa \geq \alpha_{1}+c \stackrel{\text { level }}{\geq} a+\gamma_{1} \geq \rho+\tau .
\end{array}
$$

Thus, $P(s)=H_{3}^{+}(s)<H_{2}^{-}(s) \leq N(s)$ for $s \geq t$ by Remark 4.3.

(3) $\Delta H_{1}^{-}(t)=-1$. Remark 4.3 implies that $H_{1}^{+}$has ended. We will assume that $\Delta H_{2}^{-} \neq-1$ and $\Delta H_{3}^{-} \neq-1$ since those cases are addressed above. We will look at $I_{1}$ and $I_{2}$ separately.

For $I_{1}$ we have

$$
t \geq \sigma+\tau \geq \mu+\tau \quad \Rightarrow \quad H_{2}^{-}(t) \text { has started. }
$$

If $\Delta H_{2}^{-}(t)=1$, then $N$ is not decreasing and thus cannot decrease to be less than $P$. Furthermore, $H_{2}^{-}(t)$ cannot be over, or else the whole Hilbert function is over. Thus, $\Delta H_{2}^{-}(t)=0$ so $H_{2}^{-}(t)$ is a non-zero constant. Thus, $H_{2}^{-}(s) \geq H_{2}^{+}(s)$ and $H_{1}^{-}(s)>H_{3}^{+}(s)$ (by Remark 4.3) for all $s \geq t$. This implies that $N(s) \geq H_{2}^{-}(s)+H_{1}^{-}(s)>H_{2}^{+}(s)+H_{3}^{+}(s)=P(s)$ for all $s \geq t$.

For $I_{2}$, we have

$$
\begin{gathered}
t \geq \sigma+\tau \geq \eta+\tau \quad \text { and } \quad t \geq \sigma+\tau \geq \kappa+\tau \\
\Rightarrow \Delta H_{2}^{+}(t)=-1 \quad \text { or } \quad H_{2}^{+}(t) \text { ended } \\
t \geq \sigma+\tau \geq \xi+\kappa+\tau \Rightarrow \Delta H_{3}^{+}(t) \leq 0 .
\end{gathered}
$$

Thus, if $H_{2}^{+}(t)=-1$, then $P$ is decreasing and will remain less than $N$. If $H_{2}^{+}(t)$ has ended, then $P(t)=H_{3}^{+}(t)$. Remark 4.3 implies that $H_{1}^{-}(s)>$ $H_{3}^{+}(s)$ and $N(s)>P(s)$ for $s \geq t$.

Proposition 4.4. Let

$$
I=\left(x^{a}, x^{\alpha_{2}} z^{\gamma_{1}}, x^{\alpha_{2}} y^{\beta_{1}}, z^{c}, y^{\beta_{2}} z^{\gamma_{2}}, x^{\alpha_{1}} y^{\beta_{2}}, y^{b}\right),
$$

where $a+\beta_{1}+\gamma_{1}=\alpha_{1}+b+\gamma_{2}=\alpha_{2}+\beta_{2}+c$ and $a \geq \alpha_{2} \geq \alpha_{1}, b \geq \beta_{2} \geq \beta_{1}$, $c \geq \gamma_{2} \geq \gamma_{1}$. Let $H_{R / I}$ be the Hilbert function of $R / I$. Then

$$
H_{R / I}=H\left(\alpha_{2}, \beta_{2}, c\right)+H\left(a-\alpha_{2}, \beta_{1}, \gamma_{1}\right)\left(-\alpha_{2}\right)+H\left(\alpha_{1}, b-\beta_{2}, \gamma_{2}\right)\left(-\beta_{2}\right) .
$$

This Hilbert function is strictly unimodal.

Proof. For strict unimodality, we will show that $\Delta H_{R / I}$ is positive, possibly zero, then negative. Let us first define the following variables:

$$
\begin{array}{ll}
\rho=\min \left\{\alpha_{2}, \beta_{2}\right\}, & \eta=\min \left\{a-\alpha_{2}, \beta_{1}\right\}, \quad \nu=\min \left\{\alpha_{1}, b-\beta_{2}\right\}, \\
\sigma=\max \left\{\alpha_{2}, \beta_{2}\right\}, \quad \mu=\max \left\{a-\alpha_{2}, \beta_{1}\right\}, \quad \omega=\max \left\{\alpha_{1}, b-\beta_{2}\right\} .
\end{array}
$$


Lemma 2.4 gives us the decomposition

$$
\begin{aligned}
\Delta H_{R / I}= & {\left[H(\rho, c)+H\left(\eta, \gamma_{1}\right)\left(-\alpha_{2}\right)+H\left(\nu, \gamma_{2}\right)\left(-\beta_{2}\right)\right] } \\
& -\left[H(\rho, c)(-\sigma)+H\left(\eta, \gamma_{1}\right)\left(-\mu-\alpha_{2}\right)+H\left(\nu, \gamma_{2}\right)\left(-\omega-\beta_{2}\right)\right] .
\end{aligned}
$$

For convenience, write this decomposition as

$$
\Delta H_{R / I}=\left[H_{1}^{+}+H_{2}^{+}+H_{3}^{+}\right]-\left[H_{1}^{-}+H_{2}^{-}+H_{3}^{-}\right] .
$$

This ideal requires two cases, one with $\rho=\alpha_{2} \leq \beta_{2}=\sigma$, and another with $\rho=\beta_{2}<\alpha_{2}=\sigma$. First, assume $\rho=\alpha_{2}$. Note that $H_{3}^{+}$and $H_{1}^{-}$start in the same degree. Furthermore, since

$$
\nu+\beta_{2} \leq \alpha_{1}+\beta_{2} \leq \alpha_{2}+\beta_{2} \quad \text { and } \quad \gamma_{2}+\beta_{2} \leq c+\beta_{2}
$$

we have that $H_{3}^{+}$will be constant before $H_{1}^{-}$is constant and $H_{3}^{+}$will decrease before $H_{1}^{-}$decreases. This implies that $H_{3}^{+}-H_{1}^{-} \leq 0$. Thus, we will consider $H_{1}^{-}-H_{3}^{+}$a negative segment and call it $H_{4}^{-}$. Our decomposition is now

$$
\Delta H_{R / I}=\left[H_{1}^{+}+H_{2}^{+}\right]-\left[H_{4}^{-}+H_{2}^{-}+H_{3}^{-}\right]:=P-N \text {. }
$$

To show that the Hilbert function is unimodal, it is enough to show that there do not exist integers $t_{1}<t_{2} \leq e$ such that $P\left(t_{1}\right)=N\left(t_{1}\right)$ and $P\left(t_{2}\right)>$ $N\left(t_{2}\right)$ or $P\left(t_{1}\right)<N\left(t_{1}\right)$ and $P\left(t_{2}\right) \geq N\left(t_{2}\right)$. First, we will make some remarks.

REMARK 4.5. (i) The peak (constant) value of $H_{4}^{-}$is less than or equal to the peak (constant) value of $H_{1}^{+}$since the peak value of $H_{1}^{-}$equals that of $H_{1}^{+}$, and subtracting $H_{3}^{+}$decreases it.

(ii) If $H_{4}^{-}$is decreasing, then $H_{1}^{-}$is decreasing and $H_{3}^{+}$is over. We see this since $H_{4}^{-}=H_{1}^{-}-H_{3}^{+}$, and if $H_{4}^{-}$is decreasing without $H_{1}^{-}$decreasing, then $H_{3}^{+}$is increasing. However, the inequalities from (1) imply that if $\Delta H_{3}^{+}=1$ then $\Delta H_{1}^{-}=1$. Thus, if $\Delta H_{3}^{+}=1$, then $H_{4}^{-}$is constant. Furthermore, (1) implies that when $H_{1}^{-}$is decreasing, then $H_{3}^{+}$is decreasing or over. If $H_{3}^{+}$is decreasing (with $H_{1}^{-}$decreasing), then $H_{4}^{-}$is constant. Thus for $H_{4}^{-}$to be decreasing, $H_{1}^{-}$is decreasing and $H_{3}^{+}$is over. This also implies that $H_{4}^{-}$will decrease by exactly one in each degree, since that is the rate that $H_{1}^{-}$decreases.

(iii) If $\Delta H_{i}^{-}(t)=-1$ for $i=2,3,4$, and $\Delta H_{j}^{+}(t)=-1$ for $j=1,2$ then $H_{i}^{-}(s) \geq H_{j}^{+}(s)$ for all $s \geq t$ and all $i$ and $j$. This is since each segment will continue to decrease by exactly one each degree until it ends and the levelness of the ideal forces $H_{j}^{+}$to end before $H_{i}^{-}$.

Claim 3. If $0 \neq P\left(t_{1}\right) \leq N\left(t_{1}\right)$, then $\Delta P(t) \leq 0$ for $t \geq t_{1}$.

Proof. We will prove the contrapositive, that if $\Delta P(t)>0$ then $P(s)>$ $N(s)$ for all $s \leq t$. Assume $\Delta P(t)>0$, then $\Delta H_{1}^{+}(t)>0$ or $\Delta H_{2}^{+}(t)>0$.

If $\Delta H_{1}^{+}(t)>0$ then $t \leq \rho \leq \sigma$, thus $t \leq \alpha_{2} \leq \beta_{2}$. This implies that $H_{2}^{-}, H_{3}^{-}$ and $H_{4}^{-}$have not yet started so $P(s)>N(s)$ for all $s \leq t$. 
If $\Delta H_{2}^{+}(t)>0$ then $t \leq \eta+\alpha_{2} \leq \mu+\alpha_{2}$ which implies that $H_{2}^{-}$has not yet started. Furthermore, $H_{2}^{+}(t)>H_{3}^{-}(t)$ since $H_{3}^{-}$started after $H_{2}^{+}$. We can assume that $\Delta H_{1}^{+}(t) \ngtr 0$ since that case was addressed above. If $H_{1}^{+}(t)$ is a non-zero constant, then $H_{1}^{+} \geq H_{4}^{-}$(Remark 4.5), thus $P(s)>N(s)$ for all $s \leq t$. If $\Delta H_{1}^{+}(t)=-1$ then $\Delta P(t)=0$, contradicting our initial assumption. Finally, if $H_{1}^{+}$is over in degree $t$, then $t \geq \alpha_{2}+c \geq \alpha_{2}+\gamma_{1}$ which contradicts our assumption that $\Delta H_{2}^{+}(t)>0$.

Claim 4. There do not exist degrees $t_{1}<t_{2} \leq e$ such that $P\left(t_{1}\right)=N\left(t_{1}\right)$ and $P\left(t_{2}\right)>N\left(t_{2}\right)$ or $P\left(t_{1}\right)<N\left(t_{1}\right)$ and $N\left(t_{2}\right) \leq P\left(t_{2}\right)$.

Proof. Claim 3 tells us that $\Delta P(t) \leq 0$ for $t \geq t_{1}$. Therefore, if such degrees $t_{2}, t_{1}$ exist, $N$ must decrease faster than $P$. Thus, at least one segment of $N$ is decreasing for $t \geq t_{1}$.

(1) Let $\Delta H_{2}^{-}(t)=-1$. Then

$$
t \geq \gamma_{1}+\alpha_{2}+\mu \geq \gamma_{1}+\alpha_{2}+\eta \text { and } t \geq \gamma_{1}+\mu+\alpha_{2} \geq a+\gamma_{1} \stackrel{\text { level }}{\geq} \alpha_{2}+c .
$$

Thus, $H_{2}^{+}$and $H_{1}^{+}$are over in degree $t$ and $0=P(s)<N(s)$ for $s \geq t$.

(2) Let $\Delta H_{3}^{-}(t)=-1$. Then

$$
t \geq \omega+\beta_{2}+\gamma_{2} \geq b+\gamma_{2} \stackrel{\text { level }}{\geq} \beta_{2}+c \geq \alpha_{2}+c .
$$

This implies that $H_{1}^{+}$is over in degree $t$. Furthermore,

$$
\begin{aligned}
& t \geq \nu+\omega+\beta_{2}=\alpha_{1}+b \stackrel{\text { level }}{\geq} \alpha_{2}+\beta_{2} \geq \alpha_{2}+\beta_{1} \geq \eta+\alpha_{2} \quad \text { and } \\
& t \geq \omega+\gamma_{2}+\beta_{2} \geq \alpha_{1}+\beta_{2}+\gamma_{2} \geq \alpha_{2}+\gamma_{1}
\end{aligned}
$$

give us that $\Delta H_{2}^{+}(t)=-1$ or is over in degree $t$. If $H_{2}^{+}$is over, then $0=P(s)<N(s)$ for $s \geq t$. If $\Delta H_{2}^{+}(t)=-1$, then Remark 4.5 says that $N(s) \geq H_{3}^{-}(s)>H_{2}^{+}(s)=P(s)$ for $s \geq t$.

(3) Let $\Delta H_{4}^{-}=-1$. Assume that $\Delta H_{2}^{-} \neq-1$ and $\Delta H_{3}^{-} \neq-1$ as those cases are addressed above. $\Delta H_{4}^{-}(t)=-1$ implies that $\Delta H_{1}^{-}(t)=-1$ by Remark 4.5 , thus

$$
t \geq c+\sigma=c+\beta_{2} \geq \alpha_{2}+c \geq \rho+c .
$$

This implies that $H_{1}^{+}(t)$ has ended. Furthermore,

$$
t \geq \rho+\sigma=\alpha_{2}+\beta_{2} \geq \beta_{1}+\alpha_{2} \quad \text { and } \quad t \geq c+\sigma=c+\beta_{2} \geq \alpha_{2}+\gamma_{1}
$$

give us that $\Delta H_{2}^{+}(t)=-1$ or $H_{2}^{+}(t)$ is over. If $H_{2}^{+}(t)$ is over, then $0=$ $P(s)<N(s)$ for $s \geq t$. If $\Delta H_{2}^{+}(t)=-1$, then Remark 4.5 says that $N(s) \geq$ $H_{4}^{-}(s)>H_{2}^{+}(s)=P(t)$ for $s \geq t$.

For the case with $\rho=\alpha_{2}>\beta_{2}=\sigma$, let $H_{4}^{-}=H_{1}^{-}-H_{2}^{+}$. Thus,

$$
\Delta H_{R / I}=\left[H_{1}^{+}+H_{3}^{+}\right]-\left[H_{4}^{-}+H_{2}^{-}+H_{3}^{-}\right]:=P-N .
$$


The proof mimics the above proof after appropriately swapping $\mathrm{H}_{2}$ and $\mathrm{H}_{3}$.

Proposition 4.6. Let

$$
I=\left(x^{a}, x^{\alpha_{2}} z^{\gamma_{1}}, z^{c}, y^{\beta_{1}} z^{\gamma_{2}}, x^{\alpha_{1}} y^{\beta_{2}}, x^{\alpha_{1}} y^{\beta_{1}} z^{\gamma_{1}}, y^{b}\right),
$$

where $a+\beta_{2}+\gamma_{1}=\alpha_{1}+b+\gamma_{2}=\alpha_{2}+\beta_{1}+c$ and $a \geq \alpha_{2} \geq \alpha_{2}, b \geq \beta_{2} \geq \beta_{1}$, $c \geq \gamma_{2} \geq \gamma_{1}$. Let $H_{R / I}$ be the Hilbert function of $R / I$. Then

$$
\begin{aligned}
H_{R / I}= & H\left(\alpha_{1}, b, \gamma_{1}\right)+H\left(a-\alpha_{1}, \beta_{2}, \gamma_{1}\right)\left(-\alpha_{1}\right)+H\left(\alpha_{2}, \beta_{1}, c-\gamma_{1}\right)\left(-\gamma_{1}\right) \\
& +H\left(\alpha_{1}, b-\beta_{1}, \gamma_{2}-\gamma_{1}\right)\left(-\beta_{1}-\gamma_{1}\right) .
\end{aligned}
$$

This Hilbert function is strictly unimodal.

Proof. For unimodality, we will show that $\Delta H_{R / I}$ is positive, possibly zero, then negative. Without loss of generality, assume $\alpha_{1} \leq \beta_{1}$. We can do this for if $\alpha_{1}>\beta_{1}$ then either $\gamma_{1} \geq \beta_{1}$ or $\gamma_{1}<\beta_{1}$. If $\gamma_{1} \geq \beta_{1}$, swap the variables to send $x$ to $z, z$ to $y$, and $y$ to $x$. If $\gamma_{1}<\beta_{1}$, swap the variables to send $x$ to $y, y$ to $z$, and $z$ to $x$. Both cases result in the same ideal, but with $\alpha_{1} \leq \beta_{1}$. Now let us define

$$
\begin{aligned}
& \rho=\min \left\{a-\alpha_{1}, \beta_{2}\right\}, \quad \eta=\min \left\{\alpha_{2}, c-\gamma_{1}\right\}, \quad \nu=\min \left\{b-\beta_{1}, \gamma_{2}-\gamma_{1}\right\}, \\
& \sigma=\max \left\{a-\alpha_{1}, \beta_{2}\right\}, \quad \mu=\max \left\{\alpha_{2}, c-\gamma_{1}\right\}, \quad \omega=\max \left\{b-\beta_{1}, \gamma_{2}-\gamma_{1}\right\} .
\end{aligned}
$$

Then Lemma 2.4 gives the decomposition

$$
\begin{aligned}
\Delta H_{R / I}= & {\left[H\left(\alpha_{1}, \gamma_{1}\right)+H\left(\rho, \gamma_{1}\right)\left(-\alpha_{1}\right)+H\left(\eta, \beta_{1}\right)\left(-\gamma_{1}\right)\right.} \\
& \left.+H\left(\nu, \alpha_{1}\right)\left(-\beta_{1}-\gamma_{1}\right)\right] \\
& -\left[H\left(\alpha_{1}, \gamma_{1}\right)(-b)+H\left(\rho, \gamma_{1}\right)\left(-\alpha_{1}-\sigma\right)+H\left(\eta, \beta_{1}\right)\left(-\gamma_{1}-\mu\right)\right. \\
& \left.+H\left(\nu, \alpha_{1}\right)\left(-\beta_{1}-\gamma_{1}-\omega\right)\right] \\
:= & P-N .
\end{aligned}
$$

For convenience, write this as

$$
\Delta H_{R / I}=\left[H_{1}^{+}+H_{2}^{+}+H_{3}^{+}+H_{4}^{+}\right]-\left[H_{1}^{-}+H_{2}^{-}+H_{3}^{-}+H_{4}^{-}\right] .
$$

To prove strict unimodality, we will prove two claims.

Claim 5. If $0 \neq P\left(t_{1}\right) \leq N\left(t_{1}\right)$, then $\Delta P(t) \leq 0$ for $t \geq t_{1}$.

Proof. Each segment in $P$ is a complete intersection and thus has the form given in Remark 2.5. If each $H_{i}^{+}$has not started or is increasing, then $P>N$ since $H_{i}^{+} \geq H_{i}^{-}$(or both are 0) for each $i$. Thus if $0 \neq P\left(t_{1}\right) \leq N\left(t_{1}\right)$, then at least one positive segment is either constant, decreasing or over for all $t \geq t_{1}$.

We will first check when one or more segments of $P$ are either decreasing or over for $t \geq t_{1}$. We note that if $P\left(t_{1}\right) \leq N\left(t_{1}\right)$, then we cannot have two positive segments increasing in degree $t \geq t_{1}$. To see this, look at when 
$\Delta H_{i}^{+}(t)=1$ for each $i$. If $\Delta H_{1}^{+}(t)=1$, then $t \leq \alpha_{1}$ and $t \leq \gamma_{1}$ so $H_{i}^{+}(t)$ has not yet started for $i=2,3,4$. If $H_{4}^{+}(t)=1$, then $t \geq \beta_{1}+\gamma_{1} \geq \alpha_{1}+\gamma_{1}$ implying that $\Delta H_{i}^{+}(t) \leq 0$ for $i=1,2,3$. This leaves us to check when both $\Delta H_{2}^{+}(t)=1$ and $\Delta H_{3}^{+}(t)=1$. In this case, the shifts prevent $H_{2}^{-}(t), H_{3}^{-}(t)$ and $H_{4}^{-}(t)$ from having started yet so $N=H_{1}^{-}(t)$. Since $H_{2}^{+}$is increasing in degree $t$ and $H_{2}^{+}$starts before $H_{1}^{-}\left(\alpha_{1} \leq b\right)$, we have that $P(s)>H_{2}^{+}(s) \geq H_{1}^{-}(s)=N(s)$ for all $s \leq t$. This contradicts the assumption that $P\left(t_{1}\right) \leq N\left(t_{1}\right)$.

Since no two positive segments can increase at the same time for $t \geq t_{1}$, if $\Delta P(t)>0$, at least one of the $H_{i}^{+}(t)$ is over and $\Delta H_{j}^{+}(t)=1$ for $i \neq j$. We will now check when each of the $H_{i}^{+}(t)$ are over.

(1) Let $H_{4}^{+}(t)$ be over. Then

$$
t \geq \nu+\alpha_{1}+\beta_{1}+\gamma_{1} \geq \beta_{1}+\gamma_{1} \geq \alpha_{1}+\gamma_{1} .
$$

Thus $H_{i}^{+}(t) \leq 0$ for $i=1,2,3$, so $\Delta P(t) \leq 0$ for $t \geq t_{1}$.

(2) Let $H_{3}^{+}(t)$ be over. Then

$$
\begin{aligned}
& t \geq \eta+\beta_{1}+\gamma_{1} \geq \alpha_{1}+\beta_{1}+\gamma_{1} \quad \text { or } \\
& t \geq \eta+\beta_{1}+\gamma_{1}=\beta_{1}+c \geq \beta_{1}+\gamma_{2} \geq \nu+\beta_{1}+\gamma_{1} .
\end{aligned}
$$

Thus, $\Delta H_{i}^{+}(t) \leq 0$ for $i=1,2,4$; therefore $P(t) \leq 0$ for $t \geq t_{1}$.

(3) Let $H_{2}^{+}(t)$ be over. Then

$$
t \geq \rho+\gamma_{1}+\alpha_{1} \geq \alpha_{1}+\gamma_{1} \quad \Rightarrow \quad H_{1}^{+}(t) \text { has ended. }
$$

Furthermore, if $\rho=\beta_{2}$, then $P(t) \leq 0$ for $t \geq t_{1}$ since

$$
\begin{aligned}
t \geq \alpha_{1}+\beta_{2}+\gamma_{1} \geq \alpha_{1}+\beta_{1}+\gamma_{1} & \Rightarrow \Delta H_{i}^{+}(t) \leq 0 \quad \text { for } i=3,4 \\
& \Rightarrow P(t) \leq 0
\end{aligned}
$$

Now if $\rho=a-\alpha_{1}$, then

$$
t \geq a+\gamma_{1} \geq \alpha_{2}+\gamma_{1} \geq \eta+\gamma_{1} \quad \Rightarrow \quad \Delta H_{3}^{+}(t) \leq 0 .
$$

If $P(t)>0$, then we need $\Delta H_{4}^{+}(t)=1$, so $t \geq \beta_{1}+\gamma_{1}$. Thus $\Delta H_{3}^{+}(t)=-1$ or $H_{3}^{+}(t)$ has ended. If $\Delta H_{3}^{+}(t)=-1$, then $\Delta H_{3}^{+}(t)+\Delta H_{4}^{+}(t)=0$ so $P(t) \ngtr 0$. The case where $\Delta H_{3}^{+}(t)$ is over is addressed above.

(4) Let $H_{1}^{+}(t)$ be over. From above, we can assume that $H_{i}^{+}(t)$ is not over for $i=2,3,4$. Furthermore, assume that $H_{i}^{+}(t)$ is not decreasing for any $i$ as that would imply that $\Delta P(t) \ngtr 0$ since only one segment of $P$ can increase at a time. Since $H_{1}^{+}(t)$ is over, $t \geq \alpha_{1}+\gamma_{1}$, thus $\Delta H_{2}^{+}(t)=0$, and $H_{2}^{+}(t)=\gamma_{1}$. (We have that $t \geq \alpha_{1}+\gamma_{1}$, but since $\Delta H_{2}^{+}(t) \neq-1$, we have that $t<\rho+\alpha_{1}$ and thus $H_{2}^{+}(t)$ is constant at $\gamma_{1}$.) If $\Delta P(t)>0$, then either $\Delta H_{3}^{+}(t)=1$ or $\Delta H_{4}^{+}(t)=1$; however we will see that in either case $P(s) \not \leq N(s)$ for $s \leq t$. We note that

$\Delta H_{2}^{+}(t)=0 \Rightarrow t \leq \rho+\alpha_{1} \leq \sigma+\alpha_{1} \quad \Rightarrow \quad H_{2}^{-}(t)$ has not started. 
If $\Delta H_{3}^{+}(t)=1$, then

$$
\begin{aligned}
& t \leq \eta+\gamma_{1} \leq \mu+\gamma_{1} \Rightarrow H_{3}^{-}(t) \text { has not started and } \\
& t \leq \beta_{1}+\gamma_{1} \Rightarrow H_{4}^{-}(t) \text { has not started. }
\end{aligned}
$$

If $\Delta H_{4}^{+}(t)=1$, then $t \leq \nu+\beta_{1}+\gamma_{1}$ and $t \geq \beta_{1}+\gamma_{1}$. This implies that $H_{3}^{+}(t)=0$ is at least constant and since $\Delta H_{3}^{+}(t) \neq-1$, we know $t \leq \eta+\gamma_{1}$. Thus,

$$
\begin{aligned}
& t \leq \eta+\gamma_{1} \leq \mu+\gamma_{1} \quad \Rightarrow \quad H_{3}^{-}(t) \text { has not started and } \\
& t \leq \nu+\beta_{1}+\gamma_{1} \leq \omega+\beta_{1}+\gamma_{1} \Rightarrow H_{4}^{-}(t) \text { has not started. }
\end{aligned}
$$

In both cases, we have $N(s)=H_{1}^{-}(s)$ for $s \leq t$. We note that $H_{2}^{+}$ started before $H_{1}^{-}\left(\alpha_{1} \leq b\right)$ and $H_{1}^{-}(k) \leq \gamma_{1}$ for all degrees $k$. Since $H_{3}^{+}(s) \neq 0$ or $H_{4}^{+}(s) \neq 0$, we have that $P(s)>H_{2}^{+}(s) \geq H_{1}^{-}(s)=N(s)$ for $s \leq t$, contradicting the assumption that $N\left(t_{1}\right) \leq P\left(t_{1}\right)$.

Now we will check when $P\left(t_{1}\right) \leq N\left(t_{1}\right)$ when some segment(s) of $P$ are constant in degree $t_{1}$. We will assume that no segments of $P$ are decreasing or over, as those are addressed above. Due to the shifts, each negative segment does not start until after the corresponding positive segment. $H_{1}^{+}$is the first segment to start, so if we let that segment be constant and $P\left(t_{1}\right) \leq N\left(t_{1}\right)$, then we need $H_{1}^{-}\left(t_{1}\right)$ to be constant, which implies that

$$
t_{1} \geq b+\gamma_{1} \quad \Rightarrow \quad t_{1} \geq \alpha_{1}+\gamma_{1} \quad \text { or } \quad t_{1} \geq b+\alpha_{1} \quad \Rightarrow \quad t_{1} \geq \rho+\alpha_{1} .
$$

Thus, $H_{2}^{+}\left(t_{1}\right)$ is constant or over. If $P(s)>0$ for some $s>t_{1}$, we need $H_{3}^{+}(s)$ or $H_{4}^{+}(s)$ to start. However, both cases force $H_{1}^{+}(s)$ to be decreasing or over, which is addressed above. Thus if $P\left(t_{1}\right) \leq N\left(t_{1}\right)$, then $\Delta P(t) \leq 0$ for $t \geq t_{1}$.

Claim 6. There do not exist degrees $t_{1}<t_{2} \leq e$ such that $P\left(t_{1}\right)=N\left(t_{1}\right)$ and $P\left(t_{2}\right)>N\left(t_{2}\right)$ or $P\left(t_{1}\right)<N\left(t_{1}\right)$ and $N\left(t_{2}\right) \leq P\left(t_{2}\right)$.

Proof. Claim 5 says that $P$ will not increase after $t_{1}$, thus if such a $t_{2}$ exists, then $N$ needs to decrease faster than $P$. We will examine when $\Delta H_{i}^{-}(t)=-1$ for all $i$. First, we have a remark.

Remark 4.7. If $\Delta H_{j}^{-}(t)=-1$ for some $j$, then $H_{i}^{+}(k)<H_{j}^{-}(k)$ for all $k \geq t$ and any $i$.

To see this, note that the levelness of the ideal implies that for all $i, H_{i}^{+}$ ends before $H_{2}^{-}, H_{3}^{-}$and $H_{4}^{-}$. Furthermore, $H_{i}^{+}$ends before $H_{1}^{-}$for all $i$ since $H_{1}^{-}$ends in degree $h=\alpha_{1}+b+\gamma_{1}$ and

$$
\begin{aligned}
& h \geq \alpha_{1}+b+\gamma_{1} \geq \alpha_{1}+\beta_{2}+\gamma_{1} \quad \Rightarrow \quad H_{1}^{+} \text {and } H_{2}^{+} \text {are over, } \\
& h \geq \alpha_{1}+b+\gamma_{1} \geq \alpha_{2}+\beta_{1}+\gamma_{1} \quad \Rightarrow \quad H_{3}^{+} \text {is over } \\
& h \geq \alpha_{1}+b+\gamma_{1} \geq \nu+\alpha_{1}+\beta_{1}+\gamma_{1} \quad \Rightarrow \quad H_{4}^{+} \text {is over. }
\end{aligned}
$$


Since all the $H_{i}^{+}$end before any $H_{j}^{-}$and none of the positive segments can decrease by more than one each degree, if $\Delta H_{j}^{-}(t)=-1$ then $H_{j}^{-}(k)>H_{i}^{+}(k)$ for all $i$ and all $k \geq t$.

Now let us look at each case with $t \geq t_{1}$.

(1) Let $\Delta H_{4}^{-}(t)=-1$, so $t \geq \alpha_{1}+\beta_{1}+\gamma_{1}+\omega$. This implies that all the $H_{i}^{+}(t)$ are over for all $i$; thus $0=P(s)<N(s)$ for $s \geq t$ since

$t \geq \alpha_{1}+\beta_{1}+\gamma_{1}+\omega \geq \alpha_{1}+\gamma_{1} \Rightarrow H_{1}^{+}(t)$ ended,

$t \geq \alpha_{1}+\beta_{1}+\gamma_{1}+\omega \geq \alpha_{1}+\gamma_{1}+\beta_{2} \geq \alpha_{1}+\gamma_{1}+\rho \Rightarrow H_{2}^{+}(t)$ ended,

$t \geq \alpha_{1}+\beta_{1}+\gamma_{1}+\omega \geq \alpha_{1}+b+\gamma_{1} \stackrel{\text { level }}{\geq} \alpha_{2}+\beta_{1}+\gamma_{1} \Rightarrow H_{3}^{+}(t)$ ended,

$t \geq \alpha_{1}+\beta_{1}+\gamma_{1}+\omega \geq \alpha_{1}+\beta_{1}+\gamma_{1}+\nu \quad \Rightarrow \quad H_{4}^{+}(t)$ ended.

(2) Let $\Delta H_{3}^{-}(t)=-1$, so $t \geq \alpha_{2}+c$ and $t \geq \beta_{1}+\gamma_{1}+\mu$. This gives that

$$
\begin{aligned}
& t \geq \alpha_{2}+c \geq \alpha_{1}+\gamma_{1} \Rightarrow H_{1}^{+}(t) \text { ended, } \\
& t \geq \alpha_{2}+c \stackrel{\text { level }}{\geq} a+\gamma_{1} \geq \gamma_{1}+\alpha_{1}+\rho \Rightarrow H_{2}^{+}(t) \text { ended, } \\
& t \geq \beta_{1}+\gamma_{1}+\mu \geq \beta_{1}+\gamma_{1}+\eta \Rightarrow H_{3}^{+}(t) \text { ended. }
\end{aligned}
$$

Thus, for $s \geq t, P(s)=H_{4}^{+}(s)<H_{3}^{-}(s) \leq N(s)$ by Remark 4.7.

(3) Let $\Delta H_{2}^{-}(t)=-1$, so $t \geq \rho+\alpha_{1}+\sigma$ and $t \geq \alpha_{1}+\gamma_{1}+\sigma$. Then

$t \geq \rho+\alpha_{1}+\sigma=a+\beta_{2} \stackrel{\text { level }}{\geq} \alpha_{1}+b \Rightarrow \Delta H_{1}^{-}(t) \leq 0$ or ended,

$t \geq \gamma_{1}+\alpha_{1}+\sigma \geq \gamma_{1}+\alpha_{1}+\rho \Rightarrow H_{1}^{+}(t)$ and $H_{2}^{+}(t)$ ended,

$t \geq \gamma_{1}+\alpha_{1}+\sigma \geq \gamma_{1}+a \geq \alpha_{2}+\gamma_{1} \geq \eta+\gamma_{1} \quad$ together with

$t \geq \gamma_{1}+\alpha_{1}+\sigma \geq \gamma_{1}+\alpha_{1}+\beta_{2} \geq \beta_{1}+\gamma_{1} \quad \Rightarrow \quad \Delta H_{3}^{+}(t)=-1$ or ended.

If $\Delta H_{1}^{-}(t)=-1$, then Remark 4.7 gives that $H_{3}^{+}(s)<H_{1}^{-}(s)$ and $H_{2}^{-}(s)>H_{4}^{+}(s)$ for $s \geq t$. Thus, $P(s)=H_{4}^{+}(s)+H_{3}^{+}(s)<H_{2}^{-}(s)+$ $H_{1}^{-}(s) \leq N(s)$ for $s \geq t$. If $\Delta H_{1}^{-}(t) \neq-1$, then $N$ decreases by at most one in each degree (the cases where $\Delta H_{3}^{-}(t)=-1$ and $\Delta H_{4}^{-}(t)=-1$ are addressed above). If $\Delta H_{3}^{+}(t)=-1$, then $P$ decreases by at least as much at $N$ and thus $N(s)>P(s)$. Therefore, assume that $H_{3}^{+}(t)$ has ended, and thus $P(s)=H_{4}^{+}(s)<H_{2}^{-}(s) \leq N(s)$ for $s \geq t$ by Remark 4.7.

(4) Let $\Delta H_{1}^{-}(t)=-1$, then $t \geq \alpha_{1}+b$ and $t \geq \gamma_{1}+b$. This gives us that

$t \geq \gamma_{1}+b \geq \gamma_{1}+\alpha_{1} \Rightarrow H_{1}^{+}$has ended,

$t \geq \alpha_{1}+b \geq \alpha_{1}+\beta_{2} \geq \alpha_{1}+\rho$ together with

$t \geq \gamma_{1}+b \geq \gamma_{1}+\alpha_{1} \Rightarrow \Delta H_{2}^{+}(t)=-1 \quad$ or $H_{2}^{+}$has ended,

$c \geq \gamma_{2} \quad$ with $\quad \beta_{1} \geq \alpha_{1} \quad \stackrel{\text { level }}{\Rightarrow} \quad b \geq \alpha_{2} \quad$ thus $\quad t \geq b+\gamma_{1} \geq \alpha_{2}+\gamma_{1} \geq \eta+\gamma_{1}$ with $t \geq \gamma_{1}+b \geq \gamma_{1}+\beta_{1} \Rightarrow \Delta H_{3}^{+}(t)=-1 \quad$ or $\quad H_{3}^{+}$has ended. 
Assume that $\Delta H_{i}^{-}(t) \neq-1$ for $i=2,3,4$ as that is addressed above. Thus, $N(t)$ is decreasing by at most one in each degree. If $\Delta H_{j}^{+}(t)=-1$ for any $j$, then $P$ is decreasing at the same rate or faster than $N$, so $N(t)>$ $P(t)$. Thus, assume that $H_{1}^{+}(t), H_{2}^{+}(t)$ and $H_{3}^{+}(t)$ are over so $N(s) \geqq$ $H_{1}^{-}(s)>H_{4}^{+}(s)=P(s)$ for $s \geq t$ by Remark 4.7.

\section{Licciness of the ideals}

In this section, we will use the following results of Huneke and Ulrich. Let $I=\left(x_{1}^{a_{1}}, \ldots, x_{d}^{a_{d}}\right)+I^{\#}$.

LEMMA 5.1 ([13], Lemma 2.5). Let $S=k\left[x_{1}, \ldots, x_{d}\right]$ be a polynomial ring over a field $k$, and let $I$ be an $\mathfrak{m}$-primary monomial ideal. If $I^{\#}=x^{B} K$ for some monomial $x^{B}=x_{1}^{b_{1}} \cdots x_{d}^{b_{d}}$ and a monomial ideal $K$ with $0 \neq K \neq S$, then the ideal $I^{\prime}=\left(x_{1}^{a_{1}-b_{1}}, \ldots, x_{d}^{a_{d}-b_{d}}\right)+K$ is obtained from $I$ by a double link defined by the monomial regular sequences $x_{1}^{a_{1}}, \ldots, x_{d}^{a_{d}}$ and $x_{1}^{a_{1}-b_{1}}, \ldots, x_{d}^{a_{d}-b_{d}}$.

Lemma 5.2 ([13], Lemma 2.4). Let $S=k\left[x_{1}, \ldots, x_{d}\right]$ be a polynomial ring over a field $k$, and let $I$ be an $\mathfrak{m}$-primary monomial ideal. If $I^{\#}$ has height at least two, then $I_{\mathfrak{m}}$ is not licci in $S_{\mathfrak{m}}$. In particular, I is not licci.

Now we will state and prove the main result of this section.

TheOrem 5.3. Let $a \geq \alpha_{2} \geq \alpha_{1}, b \geq \beta_{2} \geq \beta_{1}$, and $c \geq \gamma_{2} \geq \gamma_{1}$.

(1) The following ideals are licci:

(a) $\left(x^{a}, x^{\alpha_{2}} z^{\gamma_{1}}, x^{\alpha_{1}} z^{\gamma_{2}}, z^{c}, y^{\beta_{1}} z^{\gamma_{2}}, y^{\beta_{2}} z^{\gamma_{1}}, y^{b}\right)$ where $a+b+\gamma_{1}=\alpha_{2}+\beta_{2}+$ $\gamma_{2}=\alpha_{1}+\beta_{1}+c$.

(b) $\left(x^{a}, x^{\alpha_{2}} z^{\gamma_{1}}, x^{\alpha_{1}} z^{\gamma_{2}}, z^{c}, y^{\beta_{2}} z^{\gamma_{1}}, y^{b}, x^{\alpha_{1}} y^{\beta_{1}} z^{\gamma_{1}}\right)$ where $a+b+\gamma_{1}=\alpha_{2}+$ $\beta_{1}+\gamma_{2}=\alpha_{1}+\beta_{2}+c$. In particular ideals (1) and (2) from Theorem 3.1 are licci.

(2) The following ideals are not licci:

(a) $\left(x^{a}, x^{\alpha_{2}} z^{\gamma_{1}}, x^{\alpha_{2}} y^{\beta_{1}}, z^{c}, y^{\beta_{2}} z^{\gamma_{2}}, x^{\alpha_{1}} y^{\beta_{2}}, y^{b}\right)$ where $a+\beta_{1}+\gamma_{1}=\alpha_{2}+$ $\beta_{2}+c=\alpha_{1}+b+\gamma_{2}$. Assume that $c \neq \gamma_{2}$; if it does then this falls into case (1)(a) above after swapping $x$ and $z$.

(b) $\left(x^{a}, x^{\alpha_{2}} z^{\gamma_{1}}, z^{c}, y^{\beta_{1}} z^{\gamma_{2}}, y^{b}, x^{\alpha_{1}} y^{\beta_{2}}, x^{\alpha_{1}} y^{\beta_{1}} z^{\gamma_{1}}\right)$ where $a+\beta_{2}+\gamma_{1}=\alpha_{1}+$ $b+\gamma_{2}=\alpha_{2}+\beta_{1}+c$. Assume that none of the following equalities hold: $a=\alpha_{2}, b=\beta_{2}$ or $c=\gamma_{2}$. If any hold then the ideal falls into case (1)(b) above after appropriately changing the variables.

In particular, after excluding the specific cases identified above, ideals (3) and (4) in Theorem 3.1 are not licci.

Proof. For part (1), we will show that the ideals are licci by actually constructing the $C I$-links. Ideal (a) decomposes as

$$
\begin{aligned}
& I=z^{\gamma_{1}} \cdot L+\left(x^{a}, y^{b}\right) \quad \text { with } \\
& L=z^{\gamma_{2}-\gamma_{1}}\left(x^{\alpha_{1}}, y^{\beta_{1}}, z^{c-\gamma_{2}}\right)+\left(x^{\alpha_{2}}, y^{\beta_{2}}\right)=z^{\gamma_{2}-\gamma_{1}} \cdot T+J .
\end{aligned}
$$


Lemma 5.1 gives that $L$ is $C I$-linked to $T$ (a complete intersection) by the double link defined by the monomial regular sequences $C=\left(x^{\alpha_{2}}, y^{\beta_{2}}, z^{c-\gamma_{1}}\right)$ and $S=\left(x^{\alpha_{2}}, y^{\beta_{2}}, z^{c-\gamma_{2}}\right)$. Thus, $L \stackrel{C}{\sim} Y \stackrel{S}{\sim} T$ where $Y$ is some monomial ideal in $R$, so $L$ is licci. Now we will show that $I$ is in the same $C I$-liaison class as $L$ and thus it is $C I$-linked to a complete intersection. Lemma 5.1 gives that $I$ is $C I$-linked to $L$ by a double link defined by the monomial regular sequence $V=\left(x^{a}, y^{b}, z^{c}\right)$ and $X=\left(x^{a}, y^{b}, z^{c-\gamma_{1}}\right)$. Thus $I \stackrel{V}{\sim} B \stackrel{X}{\sim} L \stackrel{C}{\sim} Y \stackrel{S}{\sim} T$, where $V$ is some monomial ideal in $R$; therefore $I$ is licci.

The licciness of ideal (b) follows similarly. The ideal decomposes as

$$
\begin{aligned}
& I=z^{\gamma_{1}} \cdot L+\left(x^{a}, y^{b}\right) \quad \text { with } \\
& L=x^{\alpha_{1}}\left(x^{\alpha_{2}-\alpha_{1}}, y^{\beta_{1}}, z^{\gamma_{2}-\gamma_{1}}\right)+\left(y^{\beta_{2}}, z^{c-\gamma_{1}}\right)=x^{\alpha_{1}} \cdot T+J .
\end{aligned}
$$

Lemma 5.1 gives us that $L$ is $C I$-linked to $T$ by the double link defined by $C=\left(x^{\alpha_{2}}, y^{\beta_{2}}, z^{c-\gamma_{1}}\right)$ and $S=\left(x^{\alpha_{2}-\alpha_{1}}, y^{\beta_{2}}, z^{c-\gamma_{1}}\right)$. Thus, $L \stackrel{C}{\sim} Y \stackrel{S}{\sim} T$ where $Y$ is some monomial ideal in $R$. Furthermore, $I$ is $C I$-linked to $L$ by a double link defined by the monomial regular sequence $V=\left(x^{a}, y^{b}, z^{c}\right)$ and $X=\left(x^{a}, y^{b}, z^{c-\gamma_{1}}\right)$. Thus, we have that $I \stackrel{V}{\sim} B \stackrel{X}{\sim} L \stackrel{C}{\sim} Y \stackrel{S}{\sim} T$, where $V$ is some monomial ideal in $R$; therefore $I$ is licci.

For part (2), ideals (a) and (b) decompose as

$$
\begin{aligned}
& \left(x^{a}, y^{b}, z^{c}\right)+\left(x^{\alpha_{2}} z^{\gamma_{1}}, x^{\alpha_{2}} y^{\beta_{1}}, y^{\beta_{2}} z^{\gamma_{2}}, x^{\alpha_{1}} y^{\beta_{2}}\right) \text { and } \\
& \left(x^{a}, y^{b}, z^{c}\right)+\left(x^{\alpha_{2}} z^{\gamma_{1}}, y^{\beta_{1}} z^{\gamma_{2}}, x^{\alpha_{1}} y^{\beta_{2}}, x^{\alpha_{1}} y^{\beta_{1}} z^{\gamma_{1}}\right) \quad \text { respectively. }
\end{aligned}
$$

Since the second piece of both ideals is an ideal of height at least two, Lemma 5.2 gives us that the original ideals cannot be licci.

Conjecture 5.4. The ideals of part (2) in Theorem 5.3 are glicci ideals.

[19] and [16] have several results about the glicciness of ideals. If one can show that these ideals are generically Gorenstein, then Theorem 2.3 of [19], would apply to the ideals above and prove that they are glicci.

Acknowledgments. I would like to give special thanks to my advisor, Dr. Juan Migliore, for his patience and helpful suggestions throughout this project. I would also like to thank Dr. Uwe Nagel and Dr. Fabrizio Zanello and the referee for providing useful comments to improve the paper.

\section{REFERENCES}

[1] A. Bigatti, A. Geramita and J. Migliore, Geometric consequences of extremal behavior in a theorem of Macaulay, Trans. Amer. Math. Soc. 346 (1994), no. 1, 203-235. MR 1272673

[2] M. Boij, J. Migliore, R. Miró-Roig, U. Nagel and F. Zanello, On the shape of a pure O-sequence, Mem. Amer. Math. Soc. 218 (2012), no. 2024. MR 2931681

[3] B. Boyle, The unimodality of pure $O$-sequences of type two in four variables, to appear in Rocky Mt. J. Math. MR 3217930 
[4] H. Brenner and A. Kaid, Syzygy bundles on $\mathbb{P}$ and the weak Lefschetz property, Illinois J. Math. 51 (2007), 1299-1308. MR 2417428

[5] M. K. Chari, Two decompositions in topological combinatorics with applications to matroid complexes, Trans. Amer. Math. Soc. 349 (1997), no. 10, 3925-3943. MR 1422892

[6] D. Cook II and U. Nagel, The weak Lefschetz property, monomial ideals, and lozenges, Illinois J. Math. 55 (2011), no. 1, 377-395. MR 3006693

[7] A. V. Geramita, Inverse systems of fat points: Waring's problem, secant varieties and Veronese varieties and parametric spaces of Gorenstein ideals, The Curves Seminar at Queen's, vol. X, Queen's Papers in Pure and Appl. Math., vol. 102, 1996, pp. 3-114. MR 1381732

[8] A. V. Geramita, T. Harima, J. Migliore and Y. S. Shin, The Hilbert function of a level algebra, Mem. Amer. Math. Soc. 186 (2007), no. 872. MR 2292384

[9] A. V. Geramita and J. Migliore, A generalized liaison addition, J. Algebra 163 (1994), 139-164. MR 1257311

[10] T. Harima, J. Migliore, U. Nagel and J. Watanabe, The weak and strong Lefschetz properties for Artinian K-algebras, J. Algebra 262 (2003), 99-126. MR 1970804

[11] T. Hausel, Quaternionic geometry of matroids, Cent. Eur. J. Math. 3 (2005), no. 1, 26-38. MR 2110782

[12] T. Hibi, What can be said about pure O-sequences? J. Combin. Theory Ser. A 50 (1989), no. 2, 319-322. MR 0989204

[13] C. Huneke and B. Ulrich, Liaison of monomial ideals, Bull. Lond. Math. Soc. 39 (2007), 384-392. MR 2331565

[14] A. Iarrobino and V. Kanev, Power sums, Gorenstein algebras, and determinantal loci, Lecture Notes in Mathematics, vol. 1721, Springer, Heidelberg, 1999. MR 1735271

[15] P. Kaski, P. Östergård and R. J. Patric, Classification algorithms for codes and designs, Algorithms and Computation in Mathematics, vol. 15, Springer, Berlin, 2006. MR 2192256

[16] J. Kleppe, R. Miró-Roig, J. Migliore, U. Nagel and C. Peterson, Gorenstein liaison, complete intersection liaison invariants and unobstructedness, Mem. Amer. Math. Soc. 154 (2001), no. 732 . MR 1848976

[17] C. Merino, S. D. Noble, M. Ramírez-Ibáñez and R. Villarroel-Flores, On the structure of the h-vector of a paving matroid, European J. Combin. 33 (2012), no. 8, 1787-1799. MR 2950481

[18] J. Migliore, Introduction to liaison theory and deficiency modules, Progress in Mathematics, vol. 165, Birkhäuser, Boston, 1998. MR 1712469

[19] J. Migliore and U. Nagel, Monomial ideals and the Gorenstein liaison class of a complete intersection, Compos. Math. 133 (2002), 25-36. MR 1918287

[20] U. Nagel, Even liaison classes generated by Gorenstein linkage, J. Algebra 209 (1998), 543-584. MR 1659879

[21] S. Oh, Generalized permutohedra, h-vectors of cotransversal matroids and pure $O$ sequences, Electron. J. Combin. 20 (2013), no. 3, P14. MR 3104512

[22] L. Reid, L. Roberts and M. Roitman, On complete intersections and their Hilbert functions, Canad. Math. Bull. 34 (1991), no. 4, 525-535. MR 1136655

[23] J. Schweig, On the h-vector of a lattice path matroid, Electron. J. Combin. 17 (2010), no. 1, N3. MR 2578897

[24] R. Stanley, Cohen-Macaulay complexes, Higher combinatorics (M. Aigner, ed.), Reidel, Dordrecht, 1977, pp. 51-62. MR 0572989

[25] R. Stanley, Weyl groups, the hard Lefschetz theorem, and the Sperner property, SIAM J. Algebr. Discrete Methods 1 (1980), 168-184. MR 0578321

[26] E. Stokes, The h-vectors of 1-dimensional matroid complexes and a conjecture of Stanley, preprint; available at http://arxiv.org/abs/0903.3569. 
[27] J. Watanabe, The Dilworth number of Artinian rings and finite posets with rank function, Commutative algebra and combinatorics, Advanced Studies in Pure Math., vol. 11, Kinokuniya Co. North Holland, Amsterdam, 1987. MR 0951211

[28] F. Zanello, A non-unimodal codimesion three level h-vector, J. Algebra 305 (2006), no. 2, 949-956. MR 2266862

Bernadette Boyle, Sacred Heart University, 5151 Park Ave, Fairfield, CT 06825, USA

E-mail address: boyleb7@sacredheart.edu 